\title{
Effects of P-MAPA as immunomodulator on markers for energy metabolism in bladder cancer
}

\author{
Wagner J. Fávaro ${ }^{1 *}$, Petra K. Böckelmann ${ }^{1}$, Patrick V. Garcia, Eduardo A.R. Socca ${ }^{1}$, N. Durán ${ }^{1,2^{*}}$
}

Cite this article: Fávaro WJ, Böckelmann PK, Garcia PV, Socca EA, Durán N: Effects of P-MAPA as Immunomodulator on Markers for Energy Metabolism in Bladder Cancer. Ann Urol Oncol 2020; 3(1): 27-35. https://doi.org/10.32948/auo.2020.07.27

\begin{abstract}
Introduction This study characterized and compared cellular energetic metabolism features in the treatment of chemically induced non-muscle invasive bladder cancer (NMIBC) in Fischer 344 rats that were submitted to intravesical immunotherapies with P-MAPA (Protein aggregate magnesium-ammonium phospholinoleate-palmitoleate anhydride) and Bacillus CalmetteGuérin (BCG).

Methods Rat urinary bladder samples from Control, NMIBC (Cancer), NMIBC+BCG and NMIBC+P-MAPA groups were submitted to histopathological and western blotting analyses for the following proteins: GLUT 1, PFK, GAPDH, HADHSC, $\beta$-F1-ATPase, AMPK and mTOR. Results P-MAPA Intravesical treatment was effective in tumor regression and histologic recovery of the urinary bladder in the NMIBC. There was a significant increase in protein levels of GLUT 1, mTOR, GAPDH and HADHSC in the NMIBC+P-MAPA group. It was observed an increase in protein levels of PFK and $\beta$-F1-ATPase and a significant reduction in protein levels of AMPk in the NMIBC group.

Conclusions Our results showed that immunotherapy with P-MAPA may be an alternative in the treatment of NMIBC, especially in cases where BCG therapy failure, as evidenced by the effect of P-MAPA on tumor regression.
\end{abstract}

Key words Bladder cancer, immunomodulatory, P-MAPA, BCG

\footnotetext{
1. Laboratory of Urogenital Carcinogenesis and Immunotherapy, Department of Structural and Functional Biology, University of Campinas, Campinas, SP, Brazil.

2. Nanomedicine Research Unit (Nanomed), Federal University of ABC (UFABC), Santo André, SP, Brazil.

*Correspondence: Prof. Wagner J. Fávaro and Prof. Nelson Durán (Laboratory of Urogenital Carcinogenesis and Immunotherapy, Department of Structural and Functional Biology, University of Campinas, Campinas, SP, Brazil; Email: Prof. Wagner J. Fávaro: wjfavaro@gmail.com, Prof. Nelson Durán: nelsonduran1942@gmail.com).
} 


\section{Introduction}

The American Cancer Society estimates 81,190 new cases of Bladder Cancer (BC) in the United States in 2018 and about 17,240 deaths as a result of this disease [1].

Initial $\mathrm{BC}$ treatments involve transurethral resection of bladder tumor (TURBT) [2-4]. In patients with a high risk of NMIBC, intravesical immunotherapy with Bacillus Calmette-Guérin (BCG) may be performed after TURBT as a measure to decrease tumor progression and avoid recurrence $[5,6]$. Side effects are present in $69.5 \%$ to $91 \%$ of patients treated with BCG, ranging from mild to moderate irritative symptoms of the urinary tract to death, including systemic manifestations such us sepsis, granulomatous hepatitis, miliary pneumonitis, bone marrow involvement, soft tissue infections, vascular aneurysms, arthritis and uveitis compromising their use [7-9].

Faced with these side effects, several studies have been carried out to offer new therapeutic options for NMIBC. Among these studies, compounds that are capable of acting as Toll-like receptor agonists (TLRs) can be evaluated and developed as anticancer drugs (10). The Protein aggregate magnesium-ammonium phospholinoleatepalmitoleate anhydride (P-MAPA) acts as an agonist for TLRs 2 and 4, as well as activates p53, promoting tumor regression in NMIBC, suppressing abnormal cell proliferation and preventing tumor expansion [11-14].

Understanding how neoplastic cells are capable of ensuring a positive energy balance and associating it with anabolic processes is fundamental for the development of effective therapies to fight cancer. The study of energy metabolism aims to understand and indicate, for example, which fuel is being used preferentially, which way this fuel is participating, which are the factors that regulate or modulate the speed of these ways, among others. The energy consumption of metabolic activity in normal cells is primarily based on the oxidative phosphorylation (OXPHOS), which is efficient and generates more adenosine triphosphate (ATP) in relation to the glycolysis. In aerobic conditions, the normal cells convert glucose to pyruvate via glycolysis in the cytosol and, subsequently, to carbon dioxide in the mitochondrion [15]. In anaerobic conditions, the glycolysis is favored, and relatively little pyruvate is supplied to the oxygen consuming mitochondria [15]. This process is characterized by high glucose consumption and lactate production, regardless of the oxygen inflow [15, 16]. As all neoplastic cells are dependent on this change in metabolism, these pathways represent "checkpoints" that are susceptible to changes and attractive with regard to therapeutic targets [17, 18].

Several proteins involved in the pathways of oxidative phosphorylation and glycolysis act as markers for these processes. In this study we used Glucose transporter-1 (GLUT 1), Phosphofructokinase (PFK), glyceraldehyde 3-phosphate dehydrogenase (GAPDH) as glycolytic pathway markers and, Short chain 3-hydroxyacyl-CoA dehydrogenase (HADHSC) and Mitochondrial ATP synthase F1-beta-subunit ( $\beta$-F1-ATP synthase) as the markers of the OXPHOS pathway.

The checkpoints for glycolysis and OXPHOS are regulated by some key proteins, highlighting the AMP-dependent protein kinase (AMPK). This protein promotes cell survival in the face of metabolic stress, promoting the arrest of the cell cycle through the regulation of anabolic pathways and the promotion of catabolism [19]. On the other hand, the AMPK activator, called LKB1, is absent in many tumors, which makes these cells more sensitive to nutrient deprivation, since they are unable to react and adapt to metabolic stress [19]. AMPK also participates in the inactivation of mTOR (Mammalian Target of Rapamycin) which is one of the main regulators of protein translation and proliferation. This protein, and the mTORC1 complex that is activated, detects the nutritional status of cells. If nutrients are absent, the cells do not synthesize proteins and stop growing. However, when mTOR is hyperactivated, as in an oncogenic context by mutation of the tumor suppressor TSC (tuberous sclerosis complex), the cells become susceptible to glucose deprivation. These cells try to use nutrients for anabolic pathways as they cannot match the supply and demand for nutrients [19].

Since the cells that constitute the $\mathrm{BC}$ need adjustments in cellular energy metabolism to support uncontrolled cell proliferation $[15,16]$, therapies that may hinder or inhibit these adjustments represent a promising modality for combat of this type of tumor. Thus, we evaluated the immunotherapeutic effect of the biological response modifier P-MAPA on energy metabolism in NMIBC that were chemically induced in rats.

\section{Materials and methods}

Experimental Proceedings for NMIBC Induction and Treatment The experimental protocol (3706-1 protocol) was approved by the Ethics Committee on Animal Use (UNICAMP/Brazil) for the use and care of laboratory animals. In this study were obtained, from the University of Campinas (Brazil), 40 female rats variety Fischer 344 , weighing on average of 150 grams. Thirty female were NMIBC induced according to Garcia et al. [13] and 10 animals were not induced and considered as control group. Two weeks after the induction, the animals were subjected to the cystography examination to confirm the presence of tumor. Subsequently, all the 40 animals were divided into 4 groups (10 animals per group): Control group: received an intravesical dose of $0.3 \mathrm{ml}$ of $0.9 \%$ saline solution per week for 6 consecutive weeks; NMIBC group: received the same treatment as the control group; NMIBC + BCG Group: received an intravesical dose of $106 \mathrm{CFU}$ (40 mg) of BCG that were diluted in $0.3 \mathrm{~mL}$ of $0.9 \%$ saline solution per week for 6 consecutive weeks; NMIBC + P-MAPA Group: received an intravesical dose of $5 \mathrm{mg} / \mathrm{kg}$ of P-MAPA (Farmabrasilis, São Paulo, Brazil) dissolved in $0.3 \mathrm{~mL}$ of $0.9 \%$ saline solution per week for 6 consecutive weeks. Two weeks after the treatment, the animals were euthanized, and the urinary bladders collected and submitted to histopathological and Western blotting analyses.

\section{Histopathological Analysis}

Urinary bladder, from each group, was collected and fixed in Bouin solution, dehydrated, diaphanized and embedded in plastic polymers (Paraplast Plus, ST. Louis, MO, USA). The fragments were sectioned with a thickness of $5 \mu \mathrm{m}$, on a microtome Leica RM 2165 (Leica, Munich, Germany), stained with hematoxylin-eosin and Masson's Trichrome and photographed on the light microscope Zeiss Axiophot (Zeiss, Munich, Germany). Histopathological diagnosis of each experimental group was analysed by a Pathologist and classified as proposed by the consensus of the World Health Organization / International Society of Urological Pathology [20].

\section{Western Blotting Analysis}

The urinary bladder urothelium of all animals in each experimental group was collected, frozen and subsequently submitted to the analysis of Western Blotting. The samples were homogenized in extraction buffer (Sigma-Aldrich, St. Louis, MO, USA) and the extracts of urothelium were obtained by centrifugation. Determination of protein concentration was performed by the Bradford method and readings taken by ELISA (Multiskan Photometer FC, Standard; Thermo Fisher Scientific, USA). The corresponding 70 micrograms of protein was applied to SDS-polyacrylamide gel. After electrophoresis, the material was transferred electrically to nitrocellulose membranes. The 
Table 1. Percentage of histopathological changes in the urinary bladder of rats of the Control, NMIBC, NMIBC + BCG and NMIBC + P-MAPA groups.

\begin{tabular}{|c|c|c|c|c|}
\hline Histopathology & $\begin{array}{l}\text { Control } \\
(n=5)\end{array}$ & $\begin{array}{l}\text { NMIBC } \\
(n=5)\end{array}$ & $\begin{array}{l}\text { NMIBC + BCG } \\
(n=5)\end{array}$ & $\begin{array}{l}\text { NMIBC +P-MAPA } \\
(\mathrm{n}=5)\end{array}$ \\
\hline Normal & $05(100 \%)$ & - & $01(20 \%)$ & $02(40 \%)$ \\
\hline Flat hyperplasia & - & - & - & $02(40 \%)^{*}$ \\
\hline $\begin{array}{l}\text { Papillary Urothelial } \\
\text { Carcinoma (pTa) }\end{array}$ & - & $01(20 \%)$ & $04(80 \%)^{*}$ & $01(20 \%)$ \\
\hline $\begin{array}{l}\text { Urothelial } \\
\text { Carcinoma with } \\
\text { invasion of the } \\
\text { lamina propria } \\
\text { (pT1) }\end{array}$ & - & $04(80 \%)^{*}$ & - & - \\
\hline
\end{tabular}

*Statistical significance (proportion test, $\mathrm{P}<0.0001$ )

membranes were blocked with $3 \%$ BSA diluted in TBS-T and then incubated with primary antibodies: GLUT 1 (Santa Cruz Biotechnology, Inc., USA), PFK (LifeSpan BioSciences, Inc., EUA), GAPDH (abcam, EUA), HADHSC (Santa Cruz Biotechnology, Inc., USA), ATP synthase ( $\beta$-F1-ATPase) (MyBioSource, USA), AMPK (Santa Cruz Biotechnology, Inc., USA) and mTOR (abcam, EUA). The membranes were then incubated for $2 \mathrm{~h}$ with secondary HRP-conjugated antibodies (diluted 1:3,000 in 1\% BSA; Santa Cruz Biotechnology, Inc., Santa Cruz, CA, USA). Peroxidase activity was detected by incubation with a diaminobenzidine chromogen (Sigma Chemical Co., St Louis, USA). Western blots were run in duplicate, and urinary bladder samples were grouped from 5 animals per group for each repetition. The semi-quantitative densitometry (IOD - Integrated Optical Density) analyses of the bands were conducted using NIH ImageJ $1.47 \mathrm{v}$ software (National Institute of Health, USA. Available in: http://rsb.info.nih.gov/ij/), followed by statistical analyses. $\beta$-actin was used as endogenous positive controls for standardization of the readings of band staining intensity. The results were expressed as the mean \pm standard deviation of the ratio of each band's intensity to $\beta$-actin band intensity.

\section{Statistical Analysis}

The histopathological analyses were evaluated using the ratio test and type-I error of $5 \%$ was considered statistically significant. For analysis of Western Blotting, the quantified parameters were statistically analysed for the different experimental groups. For the statistical analyses were used the Test-T and the analyses of variance (ANOVA) followed by Tukey's post hoc test for comparison of averages. All analyses were performed with $1 \%$ significance level. The results were expressed as mean \pm standard deviation.

\section{Results}

\section{Histopathological Analysis}

The animals from Control group presented normal urothelium formed of 2-3 layers: a layer of basal cells, an intermediate cell layer and a superficial or apical layer composed of umbrella cells
(Fig. 1A, 1B, 1C; Table 1).

In contrast, animals from NMIBC group presented drastic histopathological changes. Tumor urothelial cells invading the lamina propria ( $\mathrm{pT} 1)$ and papillary urothelial carcinoma (pTa) was found in $80 \%$ and $20 \%$ of the animals, respectively (Fig. 1D, 1E, 1F; Table 1). Keratinizing squamous metaplasia was found in $60 \%$ of these animals (Fig. 1D, 1E).

The most frequent histopathological change in the urinary bladder of the NMIBC + BCG group was the papillary urothelial carcinoma (pTa) (80\%). Also, 20\% of the animals from NMIBC + BCG group presented normal urinary bladder histology (Fig. 1G, 1H, 1I; Table 1).

Animals treated with P-MAPA (NMIBC + P-MAPA group) clearly showed better histopathological recovery from the cancer state than those observed in the NMIBC + BCG group, showing flat hyperplasia (40\%) (Fig. 1L; Table 1) and papillary urothelial carcinoma (pTa) (20\%) (Table 1). Normal urothelium was observed in $40 \%$ of the animals (Fig. 1J, 1K, 1L, Table 1). Also, small areas of fibrosis were identified in all animals from this group.

\section{Western Blotting Analysis}

The NMIBC+P-PMAPA group showed the highest GLUT 1 protein levels compared to NMIBC and NMIBC + BCG groups (Fig. 2A). It was observed a reduction in GLUT 1 protein levels in the NMIBC+BCG group in relation to Control group (Fig. 2A).

There was a significant increase in $\mathrm{mTOR}$ protein levels from NMIBC+P-MAPA group in comparison to other groups analyzed (Fig. 2B). The NMIBC+BCG and Control groups showed a significant increase in $\mathrm{mTOR}$ protein levels when compared to NMIBC group (Fig. 2B).

The NMIBC group showed an increase in PFK protein levels in comparison to other groups analyzed (Fig. 2C). However, no statistical differences were found among Control, NMIBC+BCG and NMIBC+P-MAPA groups (Fig. 2C).

There was a significant increase in GAPDH protein levels from NMIBC+P-PMAPA group when compared to Control group (Fig. 2D).

The animals treated with P-MAPA (NMIBC+P-MAPA group) presented an increase in the HADHSC protein levels in 

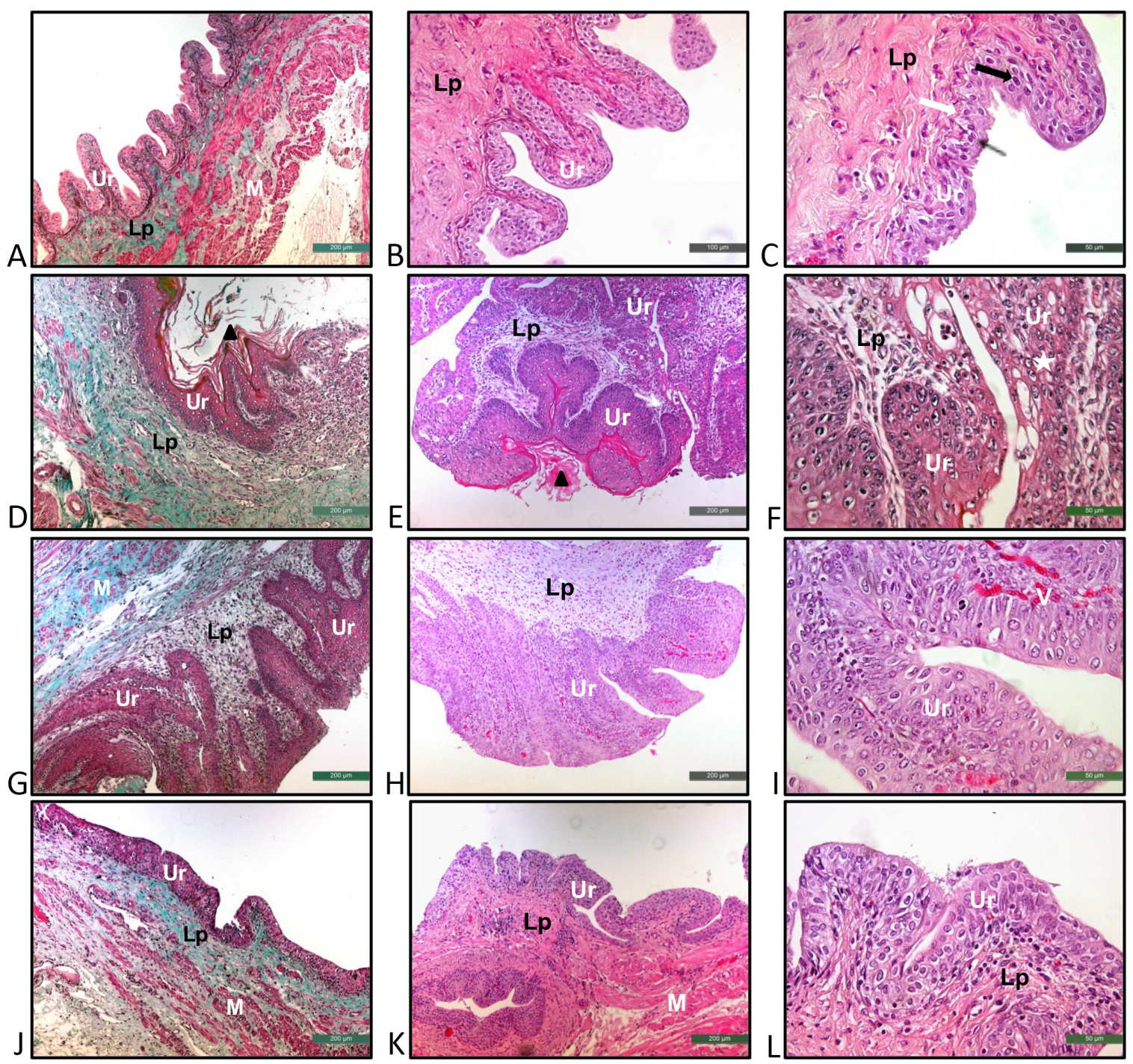

Figure 1. Representative photomicrographs stained with Masson's Trichrome (A), (D), (G), (J) and Hematoxylin-Eosin (B), (C), (E), (F), (H), (I), (K), (L) of the urinary bladders from the Control (A, B, C), NMIBC (D, E, F), NMIBC + BCG (G, H, I) and NMIBC + P-MAPA (J, K, L) groups. (A), (B), (C) Normal urothelium formed of 2-3 layers: a layer of basal cells (white arrow), an intermediate cell layer (black arrow) and a superficial layer composed of cells in umbrella (fine black arrow). (D), (E), (F) Urothelial Carcinoma with invasion of the lamina propria (pT1) (fine white arrow); keratinizing squamous metaplasia (black triangle). (G), (H), (I) Papillary urothelial carcinoma (pTa). (J), (K), (L) Flat hyperplasia. A - L: Ur - urothelium, Lp - lamina propria, M - muscular layer, Star - cellular atypia, V - blood vessels. Scale: 200 $\mu \mathrm{m}$ (A, D, E, $\mathrm{G}, \mathrm{H}, \mathrm{J}, \mathrm{K}), 100 \mu \mathrm{m}(\mathrm{B})$ and $50 \mu \mathrm{m}(\mathrm{C}, \mathrm{F}, \mathrm{I}, \mathrm{L})$.

comparison to other groups analyzed (Fig. 3A).

It was observed a significant reduction in AMPK protein levels from NMIBC+BCG group in comparison to other groups analyzed (Fig. 3B).

There was a significant increase in $\beta$-F1-ATPase protein levels from NMIBC group in comparison to other groups analyzed (Fig. 3C).

11 figures and tables should be cited in order, and also should be indicated with capital letters and bold labels, for example (Figure 1 and Table 1). Three or more figures or tables should be cited as, for example (Figure 1-3). This part can be divided into sections and described by short headings.

\section{Discussion}

Proliferating neoplastic cells exhibit considerable metabolic needs that differ from normal cells [21, 22]. In this context, it is considered that there is a metabolic heterogeneity observed in tumors influenced directly by the tumor microenvironment itself $[22,23]$. Among the many variables that may oscillate in the tumor microenvironment, abnormal tumor vascularization may result in different gradients of nutrients (glucose, amino acids and lipids), oxygen and $\mathrm{pH}$, providing the substrates for the metabolic pathways and altering the metabolism through availability of these nutrients [24].

The induction of aerobic glycolysis is one of the primary metabolic changes associated with proliferation of tumor cells. Glucose transporters (GLUTs) are responsible for glucose uptake and have tissue-specific isoforms. GLUT-1 is expressed in all tissues and in several tumors is the predominantly overexpressed isoform [25]. mTOR activation stimulates glucose uptake and also participates in the uptake of other necessary nutrients to 

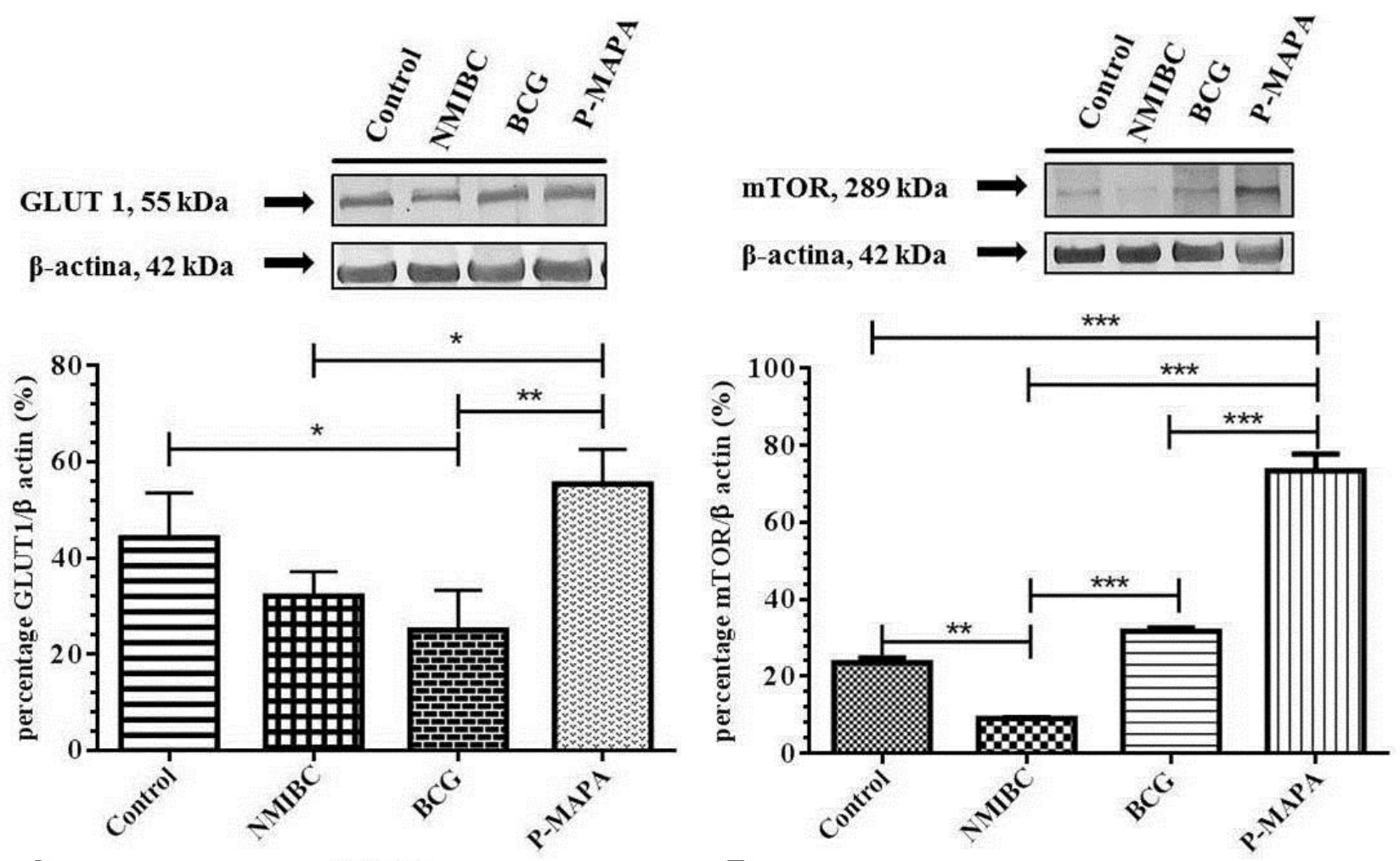

A

Groups

B

Groups
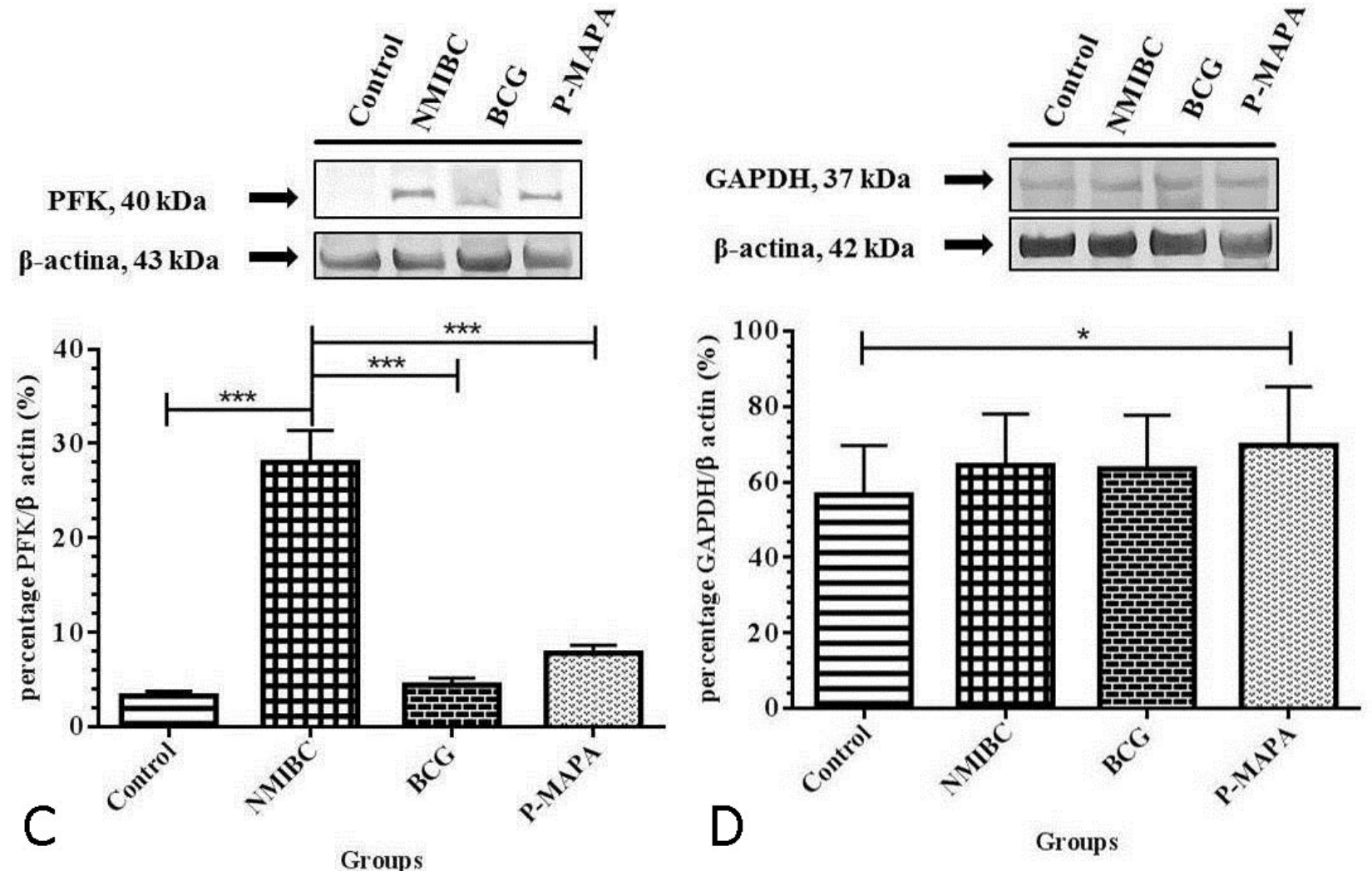

Figure 2. Representative Western blotting and semi-quantitative determination for the protein levels of GLUT 1 (A), mTOR (B), PFK (C) and GAPDH (D). The urinary bladder samples were grouped in 5 animals per group for each replicate (duplicate) and used for the semi-quantitative densitometry of GLUT1, mTOR, PFK and GAPDH levels after normalization with $\beta$-actin. All data were expressed as mean \pm standard deviation. The asterisks showed significant differences $(p<0.01)$ between the groups after the Tukey test. Experimental groups: Control, NMIBC, NMIBC + BCG (BCG), NMIBC + P-MAPA (P-MAPA). 

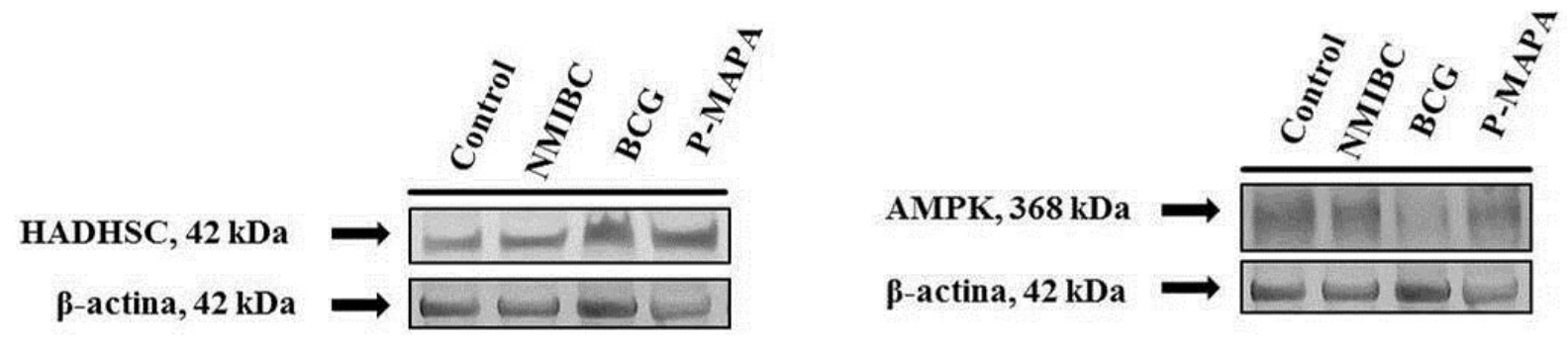

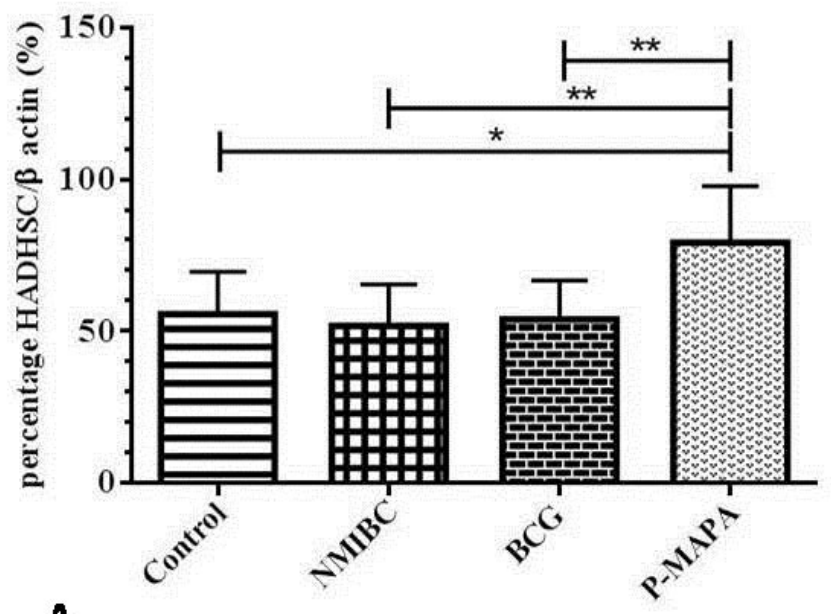

A

Groups

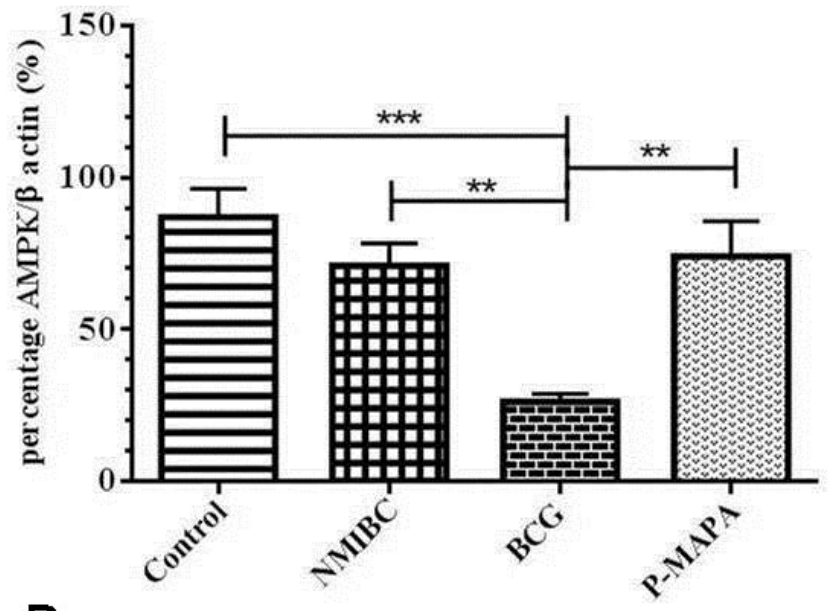

B

Groups

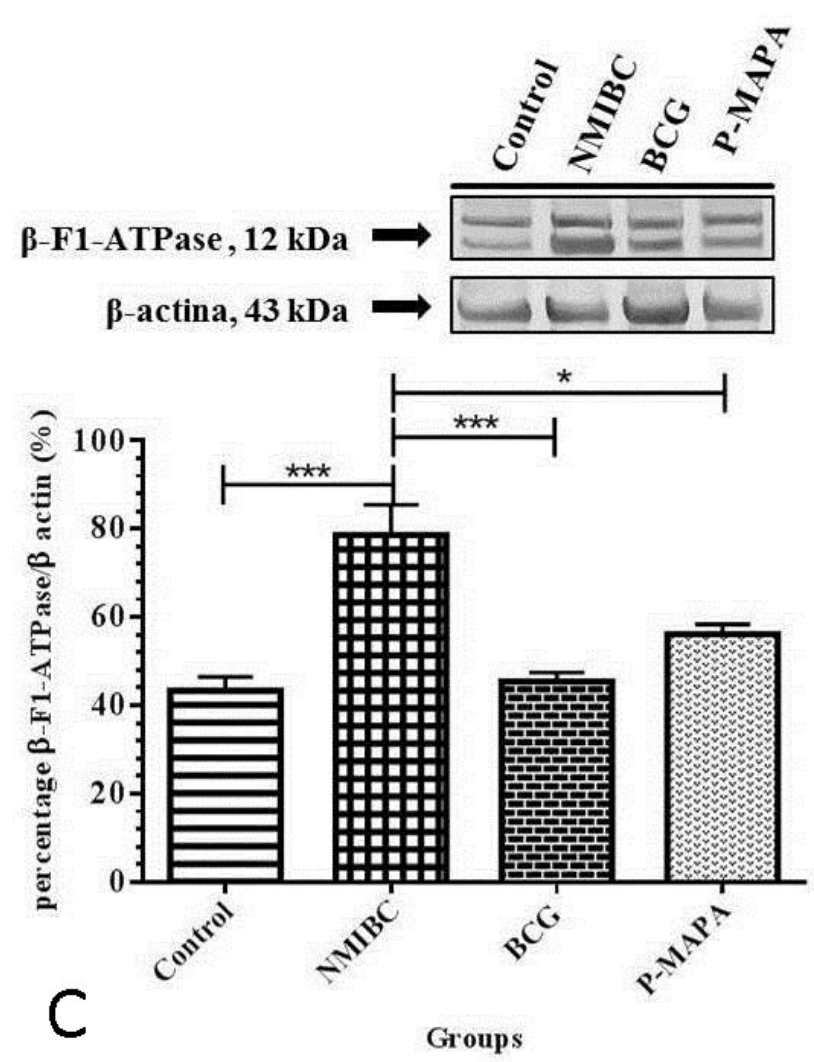

Figure 3. Representative Western blotting and semi-quantitative determination for the protein levels of HADHSC (A), AMPK (B) and $\beta$-F1ATPase (C). The urinary bladder samples were grouped in 5 animals per group for each replicate (duplicate) and used for the semi-quantitative densitometry of HADHSC, AMPK and $\beta$-F1-ATPase levels after normalization with $\beta$-actin. All data were expressed as mean \pm standard deviation. The asterisks showed significant differences $(p<0.01)$ between the groups after the Tukey test. Experimental groups: Control, NMIBC, NMIBC + BCG (BCG), NMIBC + P-MAPA (P-MAPA). 
the cell, amino acids, LDL and iron [26]. The main downstream effectors of mTOR pathway (phos S6 and 4E-BP1) have been shown to be independent predictors of prognosis in several types of solid tumors $[27,28]$. Studies demonstrated lower phos S6 and higher 4E-BP1 expression levels in urothelial tumors compared to normal urothelium $[27,28]$. The inverse correlation between phos $\mathrm{S} 6$ expression and tumor $\mathrm{pT}$ stage was related to a favorable prognostic effect of higher phos S6 levels in bladder tumors but was in contrast with prior findings of unfavorable prognostic effect of Phos S6 expression in other solid tumors [27, 28]. The downregulation of phos S6 in more aggressive bladder tumors could theoretically be related to their hypoxic tolerant phenotype given prior evidence for downregulation of mTOR pathway through HIF1 $\alpha$ activation in hypoxic states [27].

Considering the animal model of NMIBC, the present results demonstrated that the intravesical immunotherapy with P-MAPA was able to increase the GLUT-1 protein levels in relation to the animals with cancer without treatment (NMIBC group) and in relation to the animals treated with BCG (NMIBC + BCG group). This fact may be related to the increase in mTOR protein levels, which were significantly higher in the P-MAPA group in relation to these same groups (NMIBC and NMIBC + BCG groups). This increase in $\mathrm{mTOR}$ protein levels probably increased glucose uptake in animals treated with P-MAPA. This fact may be related to the mechanism of tumor urothelial cells in responding to the antiproliferative action of P-MAPA, as demonstrated in the histopathological results.

The increase in protein levels of glycolytic pathway enzymes are associated with the expression of HIF-1 $\alpha$, which is stimulated under conditions of low oxygenation, increased oxygen reactive species, cytokines and metabolic intermediates such as pyruvate, lactate and oxaloacetate [29, 30]. Inhibition or even reduction of glycolysis in tumor cells may be a useful therapeutic strategy in the fight against the disease [28]. Corroborating with these studies, our results demonstrated that the protein levels of PFK (Phosphofructokinase) in the NMIBC animal model were significantly increased. In addition, intravesical immunotherapies with P-MAPA and BCG reduced PFK levels, indicating that the reduction of these levels was an effective strategy in the treatment of NMIBC.

Interestingly, another glycolytic pathway enzyme, GAPDH, showed no significant difference in protein levels among NMIBC+BCG, NMIBC+P-MAPA and NMIBC groups. These results can be attributed to the cellular heterogeneity within the same tumor and also to the metabolic moment at which the neoplastic cells of all the analyzed groups were at the time of euthanasia and at the collection of the samples.

As the tumor cells need to adapt to the surrounding microenvironment (oxygenation, vascularization and nutrient supply) other fuel molecules, besides glucose, must participate in the maintenance of the proliferation capacity of these cells [31]. Both the lipid synthesis, regulated by the enzyme fatty acid synthase, and the oxidation of these molecules are important points for the understanding of the tumor metabolism and thus, our work evaluated the protein levels of HADHSC, participant of $\beta$-oxidation of lipids, which is responsible for the generation of ATP in healthy cells. In the animal model of NMIBC, intravesical immunotherapy with P-MAPA elevated the protein levels of HADHSC, suggesting that this immunotherapy can modify lipid metabolism, which does not seem to occur in the treatment with BCG. In addition, we suppose that P-MAPA, similar to the results found for GLUT-1, stimulated the uptake of lipids by neoplastic cells, increasing ATP as an attempt to survive the antiproliferative action performed by P-MAPA.

Furthermore, the enzyme AMPK may alter cellular energy metabolism, with consequent modification in the use of glucose for alternative substrates. For example, glioblastoma cells with active AMPK increased fatty acid oxidation by reducing lipid synthesis [32], and this increase in fatty acid oxidation appears to be related to an adaptation to energy supply (ATP) via oxidative phosphorylation (OXPHOS) to support rapid cell growth [33]. AMPK seems to function as a low energy control point, promoting various biological adjustments designed to preserve energy and adapt to metabolic changes before the cell reaches a state of energy catastrophe culminating in cell death. In our results in the NMIBC animal model, animals receiving P-MAPA intravesical immunotherapy showed increased levels of AMPK when comparing to BCG treated animals, suggesting that increased energy supply via fatty acid oxidation may be an attempt of the neoplastic cells in resisting apoptosis and the low supply of nutrients due to the suppression of angiogenesis, phenomena resulting from the action of P-MAPA [13].

The increased glycolytic pathway in the NMIBC animal model, as evidenced by the increase in PFK, seems to have been accompanied by an increase in the oxidative capacity of the cells to generate ATP, once the F1 fraction of mitochondrial ATP synthase was increased. However, according to Lopez-Rios et al. [34], tumor cell mitochondria are deficient in the $\beta$-subunit of ATPase. However, when in hypoxia, these cells accelerate the glycolytic pathway, reducing the generation of ATP through the electron transport chain [32]. This difference between the literature and the protein level of the F1 fraction of mitochondrial ATP synthase found in our results suggests that, even under stress conditions, tumor urothelial cells are still able to produce ATP by OXPHOS in this animal model. Moreover, since the most frequent histopathological alteration found in our NMIBC group results was pT $1(80 \%$ of the samples) and, since the neoplastic cells of this alteration have already invaded the lamina propria and are quite aggressive, we suggest that increased production of ATP via OXPHOS occurs because of the ability of these cells to modulate the environment to which they are inserted, increasing the supply of oxygen through the blood vessels already existing in the lamina propria, as well as through the formation of new blood vessels. In addition, our results showed that animals treated with P-MAPA had reduced protein levels of this same protein (ATP-synthase) when comparing to the levels of the NMIBC group. Garcia et al. [13] demonstrated decrease of VEGF protein levels and increase of endostatin protein levels in the NMIBC animal model treated with P-MAPA, showing the suppression of angiogenesis. This suppression of angiogenesis decreases the supply of oxygen and hampers OXPHOS.

\section{Conclusions}

Our results showed that immunotherapy with P-MAPA may be an alternative in the treatment of NMIBC, especially in cases where BCG therapy failure, as evidenced by the effect of P-MAPA on tumor regression in comparison to BCG treatment and, also the behavior of the energetic metabolism of the neoplastic cells against the action of this biological response modifier.

\section{Ethical policy}

Experimental protocol (3706-1 protocol) was approved by the Ethics Committee on Animal Use (UNICAMP/Brazil).

\section{Author contributions}

WJF coordinated all the experimental part with animals. PKB carried out the immunoassays. PVG and EARS performed the histopathological analyses. ND participated in its design and coordination and helped to draft the manuscript. All authors read 
and approved the final manuscript.

\section{Competing interests}

The authors declare no competing interests.

\section{Funding}

This research did not receive any specific grant from funding agencies in the public, commercial, or not-for-profit sectors.

\section{Acknowledgements}

Research funding for this study was supported by FarmabrasilisBrazil, NanoBioss/Sisnano (CNPq-Brazil, Process number 402280/2013-0) and FAPESP-Brazil (Process numbers 2014/11866$1 ; 2014 / 12047-4)$.

\section{References}

1. American Cancer Society. Overview: bladder cancer. In: Key statistics for bladder cancer American Cancer Society: Atlanta, GA USA.; 2018 [cited 2018 01/06/2018]. Available from: https://www. cancer.org/cancer/bladder-cancer/about/key-statistics.html.

2. Babjuk M, Böhle A, Burger M, Capoun O, Cohen D, Compérat EM, Hernandez V, Kaasinen E, Palou J, Rouorêt M et al: EAU Guidelines on non-muscle-invasive urothelial carcinoma of the bladder: update 2016. Eur Urol 2017, 71 (3): 447-461.

3. Hall MC, Chang SS, Dalbagni G, Pruthi RS, Seigne JD, Skinner EC, Wolf JS, Schellhammer PF: Guideline for the management of nonmuscle invasive bladder cancer (Stages Ta, T1, and Tis): 2007 update. J Urol 2007, 178 (6): 2314-2330.

4. Sylvester RJ: Editorial comment on: prognostic factors in patients with non-muscle-invasive bladder cancer treated with bacillus Calmette-Guerin: multivariate analysis of data from four randomized CUETO trials. Eur Urol 2008, 53 (5): 1002.

5. Ahn JJ, Ghandour RA, McKiernan JM: New agents for bacillus Calmette-Guerin-refractory nonmuscle invasive bladder cancer. Curr Opin Urol 2014, 24 (5): 540-545.

6. Buss JH, Begnini KR, Bender CB, Pohlmann AR, Guterres SS, Collares T, Seixas FK: Nano-BCG: A promising delivery system for treatment of human bladder cancer. Front Pharmacol 2018, 8 (1) : 977.

7. Pérez-Jacoiste Asín MA, Fernández-Ruiz M, López-Medrano F, Lumbreras C, Tejido A, San Juan R, Arrebola-Pajares A, Lizasoain M, Prieto S, Aguado JM: Bacillus Calmette-Guerin (BCG) infection following intravesical BCG administration as adjunctive therapy for bladder cancer incidence, risk factors, and outcome in a singleinstitution series and review of the literature. Medicine (Baltimore). 2014, 93 (17): 2236-2354.

8. Böhle A, Brandau S: Immune mechanisms in Bacillus CalmetteGuerin immunotherapy for superficial bladder cancer. J Urol 2003, 170 (3): 964-969.

9. Brausi M, Oddens J, Sylvester R, Bono A, van de Beek C, van Andel G, Gontero P, Turkeri L, Marreaud S, Coloete SW et al: Side effects of Bacillus Calmette-Guerin (BCG) in the treatment of intermediateand high-risk Ta, T1 papillary carcinoma of the bladder: results of the EORTC genito-urinary cancers group randomised phase 3 study comparing one-third dose with full dose and 1 year with 3 years of maintenance BCG. Eur Urol 2014, 65 (1): 69-76.

10. Garcia PV, Apolinário LM, Böckelmann PK, da Silva Nunes I, Durán N, Fávaro WJ: Alterations in ubiquitin ligase Siah-2 and its corepressor N-CoR after P-MAPA immunotherapy and antiandrogen therapy: new therapeutic opportunities for non-muscle invasive bladder cancer. Int J Clin Exp Pathol 2015, 8 (5): 4427-4443.
11. Durán N, Da-Silva-Nunes O: Characterization of an aggregated polymer from Penicillium sp (Pb-73 Strain). Braz J Med Biol Res 1990, 23 (12): 1289-12302.

12. Fávaro WJ, Nunes OS, Seiva FR, Nunes IS, Woolhiser LK, Durán N, Lenaerts AJ: Effects of P-MAPA immunomodulator on toll-like receptors and $\mathrm{p} 53$ : Potential therapeutic strategies for infectious diseases and cancer. Infect Agent Cancer 2012, 7 (1): 14.

13. Garcia PV, Seiva FR, Carniato AP, de Mello Júnior W, Duran N, Macedo AM, Oliveira AG, Ronih R, Iseu IS, Nunes OS et al: Increased toll-like receptors and p53 levels regulate apoptosis and angiogenesis in non-muscle invasive bladder cancer: mechanism of action of P-MAPA biological response modifier. BMC Cancer 2016, 16 (1): 422.

14. Socca EAR, Reis SK, Genaro SC, Leite SK, Reis IB, Bockelmann PK, Durán N, Fávaro WJ: P-mapa, a promisor immunomodulator against tumor cells of colonic tissues: An investigation of the action mechanism over the TLR4 signaling pathway. Life Sci 2020, 242: 117185.

15. Amoêdo ND, Valencia JP, Rodrigues MF, Galina A, Rumjanek FD: How does the metabolism of tumour cells differ from that of normal cells. Biosci Rep 2013, 33 (6): 865-873.

16. Hanahan D, Weinberg RA. Hallmarks of cancer: The next generation. Cell 2011, 144 (5): 646-674.

17. Luo J, Solimini N1, Elledge SJ: Principles of cancer therapy: oncogene and non-oncogene addiction. Cell 2009, 136 (5): 823-837.

18. Tennant DA, Duran RV, Gottlieb E: Targeting metabolic transformation for cancer therapy. Nature Rev Cancer 2010, 10: $267-$ 277.

19. Munoz-Pinedo C, El Mjiyad N, Ricci JE: Cancer metabolism: current perspectives and future directions. Cell Death Dis 2012, 3 (1): e248.

20. Epstein JI, Amin MB, Reuter VR, Mostofi FK: The World Health Organization/ International Society of Urological Pathology consensus classification of urothelial (transitional cell) neoplasms of the urinary bladder. BladderConsensus Conference Committee. Am J Surg Pathol 1998, 22 (12): 1435-1348.

21. Vander Heiden MG, Cantley LC, Thompson CB: Understanding the Warburg effect: the metabolic requirements of cell proliferation. Science 2009, 324 (5930): 1029-1033.

22. Ralph SJ, Rodríguez-Enríquez S, Neuzil J, Moreno-Sánchez R: Bioenergetic pathways in tumor mitochondria as targets for cancer therapy and the importance of the ROS-induced apoptotic trigger. Mol Aspects Med 2010, 31 (1): 29-59.

23. Cairns RA, Harris IS, Mak TW: Regulation of cancer cell metabolism. Nat Rev Cancer 2011, 11 (2): 85-95.

24. Semenza GL: HIF-1: Upstream and downstream of cancer metabolism. Curr Opin Genet Dev 2010, 20 (1): 51-56.

25. Zhao ZX, Lu LW, Qiu J, Li QP, Xu F, Liu BJ, Dong J-C, Gong W-Y: Glucose transporter-1 as an independent prognostic marker for cancer: a meta-analysis. Oncotarget 2017, 9 (2): 2728-2738.

26. Edinger AL, Thompson CB: Akt maintains cell size and survival by increasing mTOR-dependent nutrient uptake. Mol Biol Cell 2002, 13 (7): 2276-2288

27. Schultz L, Albadine R, Hicks J, Jadallah S, DeMarzo AM, Chen YB, Neilsen ME, Gonzalgo ML, Sidransky D, Schoenberg M et al: Expression status and prognostic significance of mammalian target of rapamycin pathway members in urothelial carcinoma of urinary bladder after cystectomy. Cancer 2010, 116 (23): 5517-5526.

28. Ferrari KL, de Camargo JA, Rocha GZ, Carvalheira JB, Saad MJ, Billis A, Reis, LO: Intravesical bacillus Calmette-Guérin efficiently reduces p70S6K1 but not 4E-BP1 phosphorylation in nonmuscle invasive bladder cancer. J Urol 2015, 193: 682-689.

29. Dalgard CL, Lu HS, Mohyeldin A, Verma A: Endogenous 2-oxoacids differentially regulate expression of oxygen sensors. Biochem J 2004, 380 (Pt 2): 419-424.

30. Guppy M, Leedman P, Zu X, Russell V: Contribution by different 
fuels and metabolic pathways to the total ATP turnover of proliferating MCF-7 breast cancer cells. Biochem J 2002, 364 (Pt 1): 309-315.

31. Rodríguez-Enríquez S, Marín-Hernández A, Gallardo-Pérez JC, Carreño-Fuentes L, Moreno-Sánchez R: Targeting of cancer energy metabolism. Mol Nutr Food Res 2009, 53 (1): 29-48.

32. Moreno-Sánchez R, Rodríguez-Enríquez S, Marín-Hernández A, Saavedra E: Energy metabolism in tumor cells. FEBS J 2007, 274 (6): 1393-1418.

33. Buzzai M, Bauer DE, Jones RG, DeBerardinis RJ, Hatzivassiliou G, Elstrom RL, Thompson CB.: The glucose dependence of Akttransformed cells can be reversed by pharmacologic activation of fatty acid beta-oxidation. Oncogene 2005, 24 (26): 4165-4173.

34. López-Ríos F, Sánchez-Aragó M, García-García E, Ortega AD, Berrendero JR, Pozo-Rodríguez F, López-Encuentra A, Ballestín C, Cuezva JM: Loss of the mitochondrial bioenergetic capacity underlies the glucose avidity of carcinomas. Cancer Res 2007, 67 (19): 9013-9017. 